\title{
COMBINATION OF ULTRA DOSES BUPIVACAINE PLUS FENTANYL FOR SPINAL ANESTHESIA IN OUT-PATIENT ANAL SURGERIES
}

\author{
By
Mohammad Sobhey Ibrahim Ahmed Al-Bahar, Abdulhafez Mohammad Al-Hosainy and Ahmed Mahmoud Mohamed El-Garhy
Department of Anesthesiology and Intensive Care, Faculty of Medicine, Al-Azhar University

Corresponding author: Mohammad Sobhey Ibrahim Ahmed Al-Bahar;

Mobile: 01116723370; E-mail: dr.ms.bahar3@gmail.com

\begin{abstract}
Background: Minor Anal surgeries such as piles or fissures are common problems among populations which sometimes need surgical interventions and spinal anesthesia is the optimal option for these procedures.

Objective: To assess the efficacy of combination of ultra-dose of intrathecal Bupivacaine plus fentanyl as an analgesic procedure for out-patient anal surgeries.

Patients and Methods: After approval by the local ethical committee, a prospective, controlled, clinical, randomized study was carried out on 200 patients, and randomly allocated into two equal groups: Group A: received $(2.5 \mathrm{mg})$ 0.5\% bupivacaine plus $(25 \mu \mathrm{g})$ fentanyl, and Group B: received $5 \mathrm{mg} 0.5 \%$ bupivacaine alone. An informed consent was taken from every patient subjected to this study.

Results: These studies showed that hemodynamics were more stable in (Fentanyl + Bupivacaine) group than in Bupivacaine only group. Usage of Fentanyl decreased postoperative pain and analgesic consumption in the first 6 hours after surgery along with longer pain free period compared to patients who were given Bupivacaine group.

Conclusion: Addition of $(25 \mu \mathrm{g})$ fentanyl to $(2.5 \mathrm{mg}) \quad 0.5 \%$ bupivacaine prolonged the duration of sensory spinal block, and reduced the analgesic requirement during the early post-operative period without increasing the incidence of opioid-related side-effects except pruritus, or delaying hospital discharge in patients undergoing ambulatory anorectal surgery in comparison to using $5 \mathrm{mg} 0.5 \%$ bupivacaine alone.
\end{abstract}

Keywords: Fentanyl, Bupivacaine, Anal surgeries.

\section{INTRODUCTION}

The prevalence of minor anorectal diseases in the adult population is $4-5 \%$, and approximately $10 \%$ of cases require surgical treatment. Currently, $90 \%$ of anal surgeries are performed on an ambulatory basis (Ferences, 2012). Spinal anesthesia for ambulatory surgery should be characterized by rapid onset and offset, easy administration, minimal expense, and minimal side-effects and complications (Smith, 2013).

High doses of intrathecal bupivacaine can produce extensive sensory and motor block as well as unintended prolonged arterial hypotension due to sympathetic block resulting in delayed discharge from hospital. On the other hand, low dose of 
bupivacaine is associated with a comparatively rapid recovery profile, but may not provide sufficient duration of analgesia (Maroof et al, 2015).

An alternative treatment consisting of intrathecal administration of a combination of opioids and local anesthetics produces a well-documented synergistic effect without prolonged motor nerve block or delayed discharge. Studies have shown that fentanyl in combination with low dose bupivacaine intensifies the sensory blockade and lengthens its duration without increasing the intensity of the motor blockade or prolonging recovery (Maves and Gebhart, 2012).

The present study aimed to assess the efficacy of adding Fentanyl to ultra-dose of intrathecal Bupivacaine as an analgesic procedure for out-patient anal surgeries.

\section{PATIENTS AND METHODS}

This prospective randomized singleblinded, clinical comparative study was conducted from August 2019 and ended at March 2020 in Al-Azhar University Hospitals (Al-Hussein and Sayed Galal) and approved by the ethics committee from the Department of Anesthesia of Faculty of Medicine, Al-Azhar University. Patients gave written informed consents.

The study concluded adult patients with American Society of Anesthesiologists (ASA) scoring of I -II who were underwent minor anal surgeries, e.g. hemorrhoids, anorectal fistulas, anal fissures or pilonidal sinuses, under spinal anesthesia were recruited for this study.

Patients were randomized into the two equal groups: Group A (Fentanyl group) received $(2.5 \mathrm{mg})$ of $0.5 \%$ Bupivacaine +
$(25 \mu \mathrm{g})$ Fentanyl and Group B (Bupivacaine group) received $(5 \mathrm{mg}$ ) of $0.5 \%$ Bupivacaine only.

Patients were enrolled in the study according to the following criteria: American Society of Anesthesiology grade I, II (ASA I-II), patients of either sex, aged 20 to 60 years, BMI less than 30 $\mathrm{Kg} / \mathrm{M} 2$, and scheduled for anal surgeries: piles, fissure, pilonidal sinus.

Exclusion criteria: Patient's refusal, patient in ASA groups III, IV, V, E, Body mass Index more than 30, pregnant female, abnormal coagulation profiles, skin infection, local contraindication to the technique, and patient on an analgesic regimen for any cause.

\section{Pre-operative settings:}

- Routine preoperative investigations were done to all patients including laboratory investigations as (complete blood picture, liver function tests, prothrombin time and partial thromboplastin time), chest $x$-ray and electrocardiogram. Demographic data as age, weight, and sex were recorded.

- The patients were fasting for 6 hours preoperatively. The procedure was done in the operating rooms (OR) under complete aseptic technique with prophylactic antibiotics (e.g. 2 gm ceftriaxone) 1 hour preoperatively.

Spinal anesthesia was performed at the L3 - L4 level in the sitting position using a 27-gauge Quincke needle. After free flow of cerebrospinal fluid was observed, a total volume of $1 \mathrm{ml}$ spinal solution was administered to each patient over 30 seconds. Patients were turned to the prone position immediately after the block. 
Primary outcome: Sensory and motor blockade:

a. Assessment of onset of Sensory Block: was assessed by a pin prick test Martland, et al. (2020) using a 3-point scale:

Grade 0: normal sensation.

Grade 1: decreased pain sensation to pinprick.

Grade 2: loss of pain sensation to pinprick.

The test was done every 5 minutes to loss of sensation happened and then surgical procedure started so sensation measured at zero and $5 \mathrm{~min}$. Duration of sensory block was defined as the time interval between the success of the block and the complete resolution of anesthesia.

b. Assessment of onset of Motor block:

Motor block was assessed according to the Bromage scale (Sari et al., 2015).

Duration of motor block was defined as the time interval between the success of the block and the recovery of complete motor function of forearm and hand.

\section{Secondary outcome:}

The vital signs parameters including MAP, HR and $\mathrm{SpO} 2$ were recorded at base line, 5, 10, 15, $20 \mathrm{~min}$. The assessment of postoperative pain was done with the help of Numeric Rating Scale (1-10). Zero was considered as no pain, 1-3 as mild pain, 4-6 as moderate pain and 7-10 as severe pain. At score of 4, rescue analgesic (inj. Diclofenac Sodium $(1.5 \mathrm{mg} / \mathrm{kg})$ intramuscularly) was given. Duration of analgesia will be the time from drug injection to the time of first rescue of analgesia during first 6 hours was recorded using VAS at 1, 2, 4, 6 hrs. postoperative.

Complications such as respiratory depression, nausea, vomiting and pruritus, and requests for pain relief during the early post-operative period were also noted.

\section{Statistical Analysis:}

Data were collected, revised, coded and entered to the Statistical Package for the Social Sciences (IBM SPSS) version 23. The quantitative data were presented as mean, standard deviations and ranges when parametric and median inter-quartile range (IQR) when data found nonparametric. Also qualitative variables were presented as number and percentages. The following tests were done: Independent- samples t-test of significance was used when comparing between two means. Chi-square (x2) test of significance was used in order to compare proportions between qualitative parameters. The confidence interval was set to $95 \%$ and the margin of error accepted was set to $5 \%$. P-value $<0.05$ was considered significant. 


\section{RESULTS}

There was no statistically significant difference found between group $\mathrm{A}$ and group B regarding age, gender, body mass index and total time of surgery with $\mathrm{p}$ value $=0.693,0.651,0.486$ and 0.340 respectively; while there was statistically significant difference found between the two studied groups regarding weight, height and ASA classification with pvalue $=0.011,0.008$ and 0.006 respectively (Table 1).

Table (1): Comparison between group A and group B regarding demographic data, anthropometric measures, ASA classification and total time of surgery

\begin{tabular}{|c|c|c|c|c|}
\hline \multirow{2}{*}{\multicolumn{2}{|c|}{$\begin{array}{ll}\text { Variables } & \text { Groups } \\
\end{array}$}} & Group A & Group B & \multirow{2}{*}{ P-value } \\
\hline & & No. $=100$ & No. $=100$ & \\
\hline \multirow{2}{*}{ Age } & Mean \pm SD & $28.63 \pm 4.11$ & $28.4 \pm 4.1$ & \multirow{2}{*}{0.693} \\
\hline & Range & $21-38$ & $21-36$ & \\
\hline \multirow{2}{*}{ Gender } & Females & $98(98.0 \%)$ & $97(97.0 \%)$ & \multirow{2}{*}{0.651} \\
\hline & Males & $2(2.0 \%)$ & $3(3.0 \%)$ & \\
\hline \multirow{2}{*}{ Weight } & Mean \pm SD & $73.31 \pm 6.84$ & $70.7 \pm 7.46$ & \multirow{2}{*}{0.011} \\
\hline & Range & $60-90$ & $60-90$ & \\
\hline \multirow{2}{*}{ Height } & Mean \pm SD & $171.07 \pm 6.8$ & $168.42 \pm 7.15$ & \multirow{2}{*}{0.008} \\
\hline & Range & $158-188$ & $157-188$ & \\
\hline \multirow{2}{*}{ Body mass index (BMI) } & Mean \pm SD & $25.05 \pm 1.7$ & $24.89 \pm 1.54$ & \multirow{2}{*}{0.486} \\
\hline & Range & $22-28.7$ & $22.5-29.4$ & \\
\hline \multirow{2}{*}{ ASA } & $\mathrm{I}$ & $59(59.0 \%)$ & $77(77.0 \%)$ & \multirow{2}{*}{0.006} \\
\hline & II & $41(41.0 \%)$ & $23(23.0 \%)$ & \\
\hline \multirow{2}{*}{ Total time of surgery } & Mean \pm SD & $25.55 \pm 3.25$ & $25.95 \pm 2.63$ & \multirow{2}{*}{0.340} \\
\hline & Range & $20-35$ & $20-30$ & \\
\hline
\end{tabular}

There was significant increase in motor block intensity by Bromage score in group A than group B at (zero min) and at (5 min) with $\mathrm{p}$-value $<0.001$ and $<0.001$ respectively and significant increase in sensory block in group A than group B at (zero min) with $\mathrm{p}$-value $=0.001$ while no incidence of pain found in the two studied groups at (5 $\mathrm{min}$ ) (Table 2).

Table (2): Comparison between group A and group B regarding motor block by Bromage score and sensory block by Pin Prick test score .

\begin{tabular}{|c|c|c|c|c|c|c|}
\hline \multirow[b]{2}{*}{ Parameters } & \multirow{2}{*}{ Groups } & \multicolumn{2}{|c|}{$\begin{array}{l}\text { Group A } \\
\text { No. }=\mathbf{1 0 0}\end{array}$} & \multicolumn{2}{|c|}{$\begin{array}{l}\text { Group B } \\
\text { No. }=100\end{array}$} & \multirow{2}{*}{ P-value } \\
\hline & & No. & $\%$ & No. & $\%$ & \\
\hline \multicolumn{7}{|c|}{ Motor block by Bromage score } \\
\hline \multirow{2}{*}{$\begin{array}{c}\text { zero min } \\
\text { (immediately after spinal) }\end{array}$} & 1 & 21 & $21.0 \%$ & 52 & $52.0 \%$ & \multirow{2}{*}{$<0.001$} \\
\hline & 2 & 79 & $79.0 \%$ & 48 & $48.0 \%$ & \\
\hline \multirow{2}{*}{$5 \mathrm{~min}$} & 2 & 23 & $23.0 \%$ & 80 & $80.0 \%$ & \multirow{2}{*}{$<0.001$} \\
\hline & 3 & 77 & $77.0 \%$ & 20 & $20.0 \%$ & \\
\hline \multicolumn{7}{|c|}{ Sensory block by Pin Prick test } \\
\hline \multirow{2}{*}{$\begin{array}{c}\text { zero min } \\
\text { (immediately after spinal) }\end{array}$} & Pain & 42 & $42.0 \%$ & 65 & $65.0 \%$ & \multirow{2}{*}{$<0.001$} \\
\hline & No pain & 58 & $58.0 \%$ & 35 & $35.0 \%$ & \\
\hline \multirow{2}{*}{$5 \mathrm{~min}$} & Pain & 0 & $0.0 \%$ & 0 & $0.0 \%$ & \multirow{2}{*}{1} \\
\hline & No pain & 100 & $100.0 \%$ & 100 & $100.0 \%$ & \\
\hline
\end{tabular}


There was no statistically significant difference found between group A and group B regarding use of intra-operative analgesia with $\mathrm{p}$-value $=0.088$ and significant difference found between the two studied groups regarding time of need of postoperative analgesia (hours) with $\mathrm{p}$ value $<0.001$ (Table 3).

Table (3): Comparison between group $A$ and group $B$ regarding use of intraoperative analgesia and time of need of postoperative analgesia

\begin{tabular}{|c|c|c|c|}
\hline \multirow{2}{*}{ Parameters Groups } & Group A & Group B & \multirow{2}{*}{ P-value } \\
\cline { 2 - 3 } & No. $=\mathbf{1 0 0}$ & No. = 100 & \\
\hline Intra-operative analgesia & & & \\
\hline No & $83(83.0 \%)$ & $73(73.0 \%)$ & \multirow{2}{*}{0.088} \\
\hline Yes & $17(17.0 \%)$ & $27(27.0 \%)$ & \\
\hline Postoperative need of analgesia (hrs) & & & \\
\hline Mean \pm SD & $4.02 \pm 0.56$ & $2.48 \pm 0.36$ & \multirow{2}{*}{$<0.001$} \\
\hline Range & $3.2-5$ & $2-3$ & \\
\hline
\end{tabular}

There was statistically significant difference between the two groups regarding time of need of post-operative analgesia demand at $2 \mathrm{hrs}, 4 \mathrm{hrs}$ and $6 \mathrm{hrs}$ with (Table 4)p-value $<0.001$.

Table (4): Comparison between group $A$ and group $B$ regarding post operative pain measurement at 1, 2, 4, $6 \mathrm{hrs}$ and time of need of postoperative analgesia

\begin{tabular}{|c|c|c|c|}
\hline 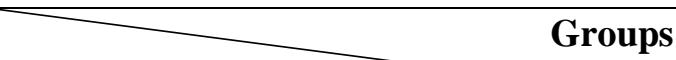 & Group A & Group B & \multirow{2}{*}{ P-value } \\
\hline Time of Post. OPAnalgesia & No. $=100$ & No. $=100$ & \\
\hline $1 \mathrm{hr}$ & $0(0.0 \%)$ & $0(0.0 \%)$ & \multirow{4}{*}{$<0.001$} \\
\hline $2 \mathrm{hrs}$ & $0(0.0 \%)$ & $48(48.0 \%)$ & \\
\hline $4 \mathrm{hrs}$ & $32(32.0 \%)$ & $52(52.0 \%)$ & \\
\hline $6 \mathrm{hrs}$ & $68(68.0 \%)$ & $0(0.0 \%)$ & \\
\hline
\end{tabular}

Systolic blood pressure was found better in hemodynamic stability in group A than group B at baseline, immediately after spinal, (10 min), (15 min), (20 min), at end of surgery and at (1 hour ) after operation with p-value $<0.001,<0.001$, <
$0.001,<0.001,<0.001,<0.001$ and $<0.001$ respectively while no statistically significant difference found between the two studied groups at $(5 \mathrm{~min})$ after induction with $\mathrm{p}$-value $=0.140$ (Table 5). 
Table (5): Comparison between group $A$ and group $B$ regarding systolic blood pressure at different times of measurement

\begin{tabular}{|c|c|c|c|c|}
\hline \multirow{2}{*}{\multicolumn{2}{|c|}{$\begin{array}{ll}\text { SBP } & \text { Groups } \\
\end{array}$}} & \multirow{2}{*}{$\begin{array}{c}\text { Group A } \\
\text { No. }=100\end{array}$} & \multirow{2}{*}{$\begin{array}{c}\text { Group B } \\
\text { No. }=100\end{array}$} & \multirow{2}{*}{ P-value } \\
\hline & & & & \\
\hline \multirow{2}{*}{ Baseline } & Mean \pm SD & $129.41 \pm 5.69$ & $125.62 \pm 8.48$ & \multirow{2}{*}{$<0.001$} \\
\hline & Range & $120-140$ & $110-140$ & \\
\hline \multirow{2}{*}{$\begin{array}{l}\text { Immediately } \\
\text { after spinal }\end{array}$} & Mean \pm SD & $122.48 \pm 4.71$ & $114.7 \pm 11.98$ & \multirow{2}{*}{$<0.001$} \\
\hline & Range & $114-132$ & $85-130$ & \\
\hline \multirow{2}{*}{$5 \mathrm{Min}$} & Mean \pm SD & $114.42 \pm 6.89$ & $112.75 \pm 8.91$ & \multirow{2}{*}{0.140} \\
\hline & Range & $95-125$ & $90-128$ & \\
\hline \multirow{2}{*}{$10 \mathrm{Min}$} & Mean \pm SD & $117.66 \pm 4.78$ & $113.61 \pm 5.42$ & \multirow{2}{*}{$<0.001$} \\
\hline & Range & $105-128$ & $105-125$ & \\
\hline \multirow{2}{*}{15 Min } & Mean \pm SD & $121.59 \pm 4.16$ & $114.72 \pm 4.65$ & \multirow{2}{*}{$<0.001$} \\
\hline & Range & $112-130$ & $105-125$ & \\
\hline \multirow{2}{*}{20 Min } & Mean \pm SD & $123.25 \pm 3.97$ & $118.96 \pm 4.36$ & \multirow{2}{*}{$<0.001$} \\
\hline & Range & $116-135$ & $110-128$ & \\
\hline \multirow{2}{*}{ End of surgery } & Mean \pm SD & $127.51 \pm 4.27$ & $123.35 \pm 6.71$ & \multirow{2}{*}{$<0.001$} \\
\hline & Range & $118-135$ & $110-134$ & \\
\hline \multirow{2}{*}{$\begin{array}{l}1 \text { hour after } \\
\text { operation }\end{array}$} & Mean \pm SD & $125.53 \pm 4.55$ & $123.03 \pm 6.52$ & \multirow{2}{*}{$<0.001$} \\
\hline & Range & $116-135$ & $110-134$ & \\
\hline
\end{tabular}

There was no statistically significant difference found between group $\mathrm{A}$ and group B regarding diastolic blood pressure at baseline and immediately after spinal with p-value $=0.067$ and 0.603 respectively. Also DBP was found better in hemodynamic stability in group B than group A at (5 min) after induction with p- value $=0.030$, while at $(10 \mathrm{~min}),(15 \mathrm{~min})$, (20 min), at end of surgery and at (1 hour) after operation the diastolic blood pressure was found better in hemodynamic stability in group A than group B with p-value < $0.001,<0.001,<0.001,<0.001$ and $<$ 0.001 respectively (Table 6).

Table (6): Comparison between group $A$ and group $B$ regarding diastolic blood pressure at different times of measurement

\begin{tabular}{|c|c|c|c|c|}
\hline \multirow{2}{*}{\multicolumn{2}{|c|}{$\begin{array}{ll}\text { DBP } & \text { Groups } \\
\end{array}$}} & Group A & Group B & \multirow{2}{*}{ P-value } \\
\hline & & No. $=100$ & No. $=100$ & \\
\hline \multirow{2}{*}{ Baseline } & Mean \pm SD & $80.09 \pm 6.6$ & $78.43 \pm 6.16$ & \multirow{2}{*}{0.067} \\
\hline & Range & $69-90$ & $68-90$ & \\
\hline \multirow{2}{*}{ Immediately after spinal } & Mean \pm SD & $72.97 \pm 5.97$ & $72.37 \pm 9.87$ & \multirow{2}{*}{0.603} \\
\hline & Range & $60-84$ & $50-88$ & \\
\hline \multirow{2}{*}{$5 \mathrm{Min}$} & Mean \pm SD & $68.82 \pm 7.28$ & $70.98 \pm 6.67$ & \multirow{2}{*}{$<0.001$} \\
\hline & Range & $50-80$ & $60-80$ & \\
\hline \multirow{2}{*}{$10 \mathrm{Min}$} & Mean \pm SD & $75.45 \pm 4.19$ & $71.56 \pm 5.36$ & \multirow{2}{*}{$<0.001$} \\
\hline & Range & $68-82$ & $60-82$ & \\
\hline \multirow{2}{*}{15 Min } & Mean \pm SD & $79 \pm 4.34$ & $71.53 \pm 4.83$ & \multirow{2}{*}{$<0.001$} \\
\hline & Range & $70-88$ & $60-80$ & \\
\hline \multirow{2}{*}{$20 \mathrm{Min}$} & Mean \pm SD & $78.63 \pm 4.62$ & $73.3 \pm 4.96$ & \multirow{2}{*}{$<0.001$} \\
\hline & Range & $70-90$ & $60-80$ & \\
\hline \multirow{2}{*}{ End of surgery } & Mean \pm SD & $78.87 \pm 4.28$ & $75.85 \pm 5.36$ & \multirow{2}{*}{$<0.001$} \\
\hline & Range & $70-90$ & $66-88$ & \\
\hline \multirow{2}{*}{1 hour after operation } & Mean \pm SD & $79 \pm 4.34$ & $71.53 \pm 4.83$ & \multirow{2}{*}{$<0.001$} \\
\hline & Range & $70-88$ & $60-80$ & \\
\hline
\end{tabular}


The mean arterial blood pressure was found better in hemodynamic stability in group A than group B at baseline, immediately after spinal, (10 $\mathrm{min}),(15$ $\mathrm{min}),(20 \mathrm{~min})$, at end of surgery and at (1 hour) after operation with $p$-value $=0.003$,
$<0.006,<0.001,<0.001,<0.001$ and $<$ 0.001 respectively while no statistically significant difference found between the two studied groups at $(5 \mathrm{~min})$ after induction with $\mathrm{p}$-value $=0.361$ (Table 7).

Table (7): Comparison between group A and group B regarding mean arterial blood pressure at different times of measurement

\begin{tabular}{|c|c|c|c|c|}
\hline \multirow{2}{*}{\multicolumn{2}{|c|}{$\begin{array}{ll}\text { MABP } & \text { Groups } \\
\end{array}$}} & \multirow{2}{*}{$\begin{array}{c}\text { Group A } \\
\text { No. }=100\end{array}$} & \multirow{2}{*}{$\begin{array}{c}\text { Group B } \\
\text { No. }=100\end{array}$} & \multirow{2}{*}{ P-value } \\
\hline & & & & \\
\hline \multirow{2}{*}{ Baseline } & Mean \pm SD & $96.6 \pm 4.98$ & $94.38 \pm 5.19$ & \multirow{2}{*}{0.003} \\
\hline & Range & $87-106$ & $84-107$ & \\
\hline \multirow{2}{*}{ Immediately after spinal } & Mean \pm SD & $89.38 \pm 4.52$ & $86.46 \pm 9.43$ & \multirow{2}{*}{$<0.006$} \\
\hline & Range & $80-97$ & $62-102$ & \\
\hline \multirow{2}{*}{5 Min } & Mean \pm SD & $84.14 \pm 6.03$ & $84.92 \pm 6.01$ & \multirow{2}{*}{0.361} \\
\hline & Range & $70-93$ & $70-95$ & \\
\hline \multirow{2}{*}{$10 \mathrm{Min}$} & Mean \pm SD & $89.52 \pm 3.67$ & $85.59 \pm 3.37$ & \multirow{2}{*}{$<0.001$} \\
\hline & Range & $80-96$ & $77-92$ & \\
\hline \multirow{2}{*}{15 Min } & Mean \pm SD & $93.11 \pm 3.54$ & $85.88 \pm 3.44$ & \multirow{2}{*}{$<0.001$} \\
\hline & Range & $84-100$ & $78-92$ & \\
\hline \multirow{2}{*}{20 Min } & Mean \pm SD & $93.5 \pm 3.25$ & $88.4 \pm 3.54$ & \multirow{2}{*}{$<0.001$} \\
\hline & Range & $87-100$ & $81-94$ & \\
\hline \multirow{2}{*}{ End of surgery } & Mean \pm SD & $95.11 \pm 3.53$ & $92.47 \pm 4.28$ & \multirow{2}{*}{$<0.001$} \\
\hline & Range & $87-105$ & $86-102$ & \\
\hline \multirow{2}{*}{1 hour after operation } & Mean \pm SD & $93.11 \pm 3.54$ & $85.88 \pm 3.44$ & \multirow{2}{*}{$<0.001$} \\
\hline & Range & $84-100$ & $78-92$ & \\
\hline
\end{tabular}

There was no statistically significant difference found between group $\mathrm{A}$ and group $\mathrm{B}$ regarding $\mathrm{SaO} 2$ at different times of measurement (Table 8).

Table (8): Comparison between group $A$ and group $B$ regarding $\mathrm{SaO} 2$ at different times of measurement

\begin{tabular}{|c|c|c|c|c|}
\hline \multirow{2}{*}{\multicolumn{2}{|c|}{ Groups }} & Group A & Group B & \multirow{2}{*}{ P-value } \\
\hline & & No. $=100$ & No. $=100$ & \\
\hline \multirow{2}{*}{ Baseline } & Mean \pm SD & $99.16 \pm 0.93$ & $99.17 \pm 0.75$ & \multirow{2}{*}{0.933} \\
\hline & Range & $97-100$ & $98-100$ & \\
\hline \multirow{2}{*}{ Immediately after spinal } & Mean \pm SD & $99.07 \pm 0.77$ & $98.88 \pm 0.82$ & \multirow{2}{*}{0.093} \\
\hline & Range & $98-100$ & $97-100$ & \\
\hline \multirow{2}{*}{5 Min } & Mean \pm SD & $98.92 \pm 0.66$ & $98.9 \pm 0.67$ & \multirow{2}{*}{0.833} \\
\hline & Range & $98-100$ & $98-100$ & \\
\hline \multirow{2}{*}{$10 \mathrm{Min}$} & Mean \pm SD & $98.95 \pm 0.80$ & $99.13 \pm 0.84$ & \multirow{2}{*}{0.121} \\
\hline & Range & $97-100$ & $97-100$ & \\
\hline \multirow{2}{*}{15 Min } & Mean \pm SD & $98.99 \pm 0.73$ & $98.86 \pm 0.65$ & \multirow{2}{*}{0.186} \\
\hline & Range & $98-100$ & $98-100$ & \\
\hline \multirow{2}{*}{$20 \mathrm{Min}$} & Mean \pm SD & $98.87 \pm 0.81$ & $98.93 \pm 0.66$ & \multirow{2}{*}{0.566} \\
\hline & Range & $98-100$ & $98-100$ & \\
\hline \multirow{2}{*}{ End of surgery } & Mean \pm SD & $98.77 \pm 0.74$ & $98.92 \pm 0.71$ & \multirow{2}{*}{0.143} \\
\hline & Range & $98-100$ & $98-100$ & \\
\hline \multirow{2}{*}{1 hour after operation } & Mean \pm SD & $99 \pm 0.64$ & $99.01 \pm 0.64$ & \multirow{2}{*}{0.912} \\
\hline & Range & $98-100$ & $98-100$ & \\
\hline
\end{tabular}


There was no statistically significant difference found between group $\mathrm{A}$ and group B regarding heart rate at different times of measurement except immediately after spinal and at end of surgery the heart rate was found better in group A than group B with p-value $<0.001$ and $<0.001$ respectively (Table 9).

Table (9): Comparison between group $A$ and group $B$ regarding heart rate at different times of measurement

\begin{tabular}{|c|c|c|c|c|}
\hline \multirow{2}{*}{\multicolumn{2}{|c|}{$\begin{array}{ll}\text { Heart Rate } & \text { Groups } \\
\end{array}$}} & \multirow{2}{*}{$\begin{array}{c}\text { Group A } \\
\text { No. }=100 \\
\end{array}$} & \multirow{2}{*}{$\begin{array}{c}\text { Group B } \\
\text { No. }=100\end{array}$} & \multirow{2}{*}{ P-value } \\
\hline & & & & \\
\hline \multirow{2}{*}{ Baseline } & Mean \pm SD & $84.71 \pm 5.63$ & $84.33 \pm 7.06$ & \multirow{2}{*}{0.674} \\
\hline & Range & $70-97$ & $70-98$ & \\
\hline \multirow{2}{*}{ Immediately after spinal } & Mean \pm SD & $85.69 \pm 8.25$ & $79.29 \pm 15.84$ & \multirow{2}{*}{$<0.001$} \\
\hline & Range & $60-97$ & $45-98$ & \\
\hline \multirow{2}{*}{5 Min } & Mean \pm SD & $84.1 \pm 10.31$ & $83.62 \pm 16.3$ & \multirow{2}{*}{0.804} \\
\hline & Range & $48-97$ & $44-115$ & \\
\hline \multirow{2}{*}{$10 \mathrm{Min}$} & Mean \pm SD & $87.62 \pm 7.96$ & $89.06 \pm 11.31$ & \multirow{2}{*}{0.299} \\
\hline & Range & $70-110$ & $70-120$ & \\
\hline \multirow{2}{*}{15 Min } & Mean \pm SD & $86.3 \pm 6.21$ & $86.52 \pm 7.43$ & \multirow{2}{*}{0.821} \\
\hline & Range & $70-97$ & $70-105$ & \\
\hline \multirow{2}{*}{$20 \mathrm{Min}$} & Mean \pm SD & $85.67 \pm 5.77$ & $86.8 \pm 6.22$ & \multirow{2}{*}{0.185} \\
\hline & Range & $70-97$ & $70-97$ & \\
\hline \multirow{2}{*}{ End of surgery } & Mean \pm SD & $86.99 \pm 5.56$ & $83.01 \pm 6.53$ & \multirow{2}{*}{$<0.001$} \\
\hline & Range & $75-97$ & $70-94$ & \\
\hline \multirow{2}{*}{1 hour after operation } & Mean \pm SD & $85.67 \pm 5.77$ & $86.8 \pm 6.22$ & \multirow{2}{*}{0.185} \\
\hline & Range & $70-97$ & $70-97$ & \\
\hline
\end{tabular}

That there was no incidence of respiratory depression and ECG changes was found in both groups; also the table shows that the incidence of pruritits was found higher in group A than group B with p-value $<0.001$; also the incidence of nausea and vomiting was found higher in group $\mathrm{B}$ than group $\mathrm{A}$ with $\mathrm{p}$-value = 0.004 and $<0.001$ respectively (Table 10).

Table (10): Comparison between group A and group B regarding complications of spinal anesthesia

\begin{tabular}{|c|c|c|c|c|}
\hline \multirow{2}{*}{\multicolumn{2}{|c|}{$\begin{array}{ll}\text { Complications } & \text { Groups } \\
\end{array}$}} & \multirow{2}{*}{$\begin{array}{c}\text { Group A } \\
\text { No. }=100 \\
\end{array}$} & \multirow{2}{*}{$\begin{array}{c}\text { Group B } \\
\text { No. }=100 \\
\end{array}$} & \multirow{2}{*}{ P-value } \\
\hline & & & & \\
\hline \multirow{2}{*}{ Respiratory depression } & No & $100(100.0 \%)$ & $100(100.0 \%)$ & \multirow{2}{*}{1} \\
\hline & Yes & $0(0.0 \%)$ & $0(0.0 \%)$ & \\
\hline \multirow{2}{*}{ ECG changes } & No & $100(100.0 \%)$ & $100(100.0 \%)$ & \multirow{2}{*}{1} \\
\hline & Yes & $0(0.0 \%)$ & $0(0.0 \%)$ & \\
\hline \multirow{2}{*}{ Pruritis } & No & $73(73.0 \%)$ & $92(92.0 \%)$ & \multirow{2}{*}{$<0.001$} \\
\hline & Yes & $27(27.0 \%)$ & $8(8.0 \%)$ & \\
\hline \multirow{2}{*}{ Nausea } & No & $92(92.0 \%)$ & $77(77.0 \%)$ & \multirow{2}{*}{0.004} \\
\hline & Yes & $8(8.0 \%)$ & $23(23.0 \%)$ & \\
\hline \multirow{2}{*}{ Vomiting } & No & $100(100.0 \%)$ & $87(87.0 \%)$ & \multirow{2}{*}{$<0.001$} \\
\hline & Yes & $0(0.0 \%)$ & $13(13.0 \%)$ & \\
\hline
\end{tabular}




\section{DISCUSSION}

The results of the present study indicated that for out-patient anorectal surgery, intrathecal administration of (25 $\mu \mathrm{g})$ fentanyl combined with an ultra-low dose of bupivacaine provides good-quality spinal anesthesia and reduces the need for early post-operative analgesic supplementation. Furthermore, this protocol was well suited for the outpatient setting because it is associated with rapid recovery of full motor power, sensory function and less side effects. This suggests a potential synergism between fentanyl and bupivacaine.

The intrathecal administration of opioids selectively decreases nociceptive afferent input from $\mathrm{A} \delta$ and $\mathrm{C}$ fibers without affecting dorsal root axons or somatosensory evoked potentials (Gurbet et al., 2018).

Lipophilic opioids, such as fentanyl, have a favorable clinical profile with fast onset, modest duration $(1-4 \mathrm{~h})$ and little risk of delayed respiratory depression (Mehta, 2020).

The recommended safe effective dose of intrathecal fentanyl is $(10-25 \mu \mathrm{g})$. Numerous clinical studies have demonstrated that intrathecal fentanyl does not prolong the duration of motor blockade (Gupta et al., 2013).

In an attempt to modify anesthesia for ambulatory surgery, several investigators have evaluated intrathecal fentanyl in combination with smaller doses of spinal local anesthetic. In a randomized, doubleblind study involving gynecological laparoscopy Kendall et al. (2018).

They found improved intraoperative analgesia and prolonged sensory block, but no difference in motor recovery or time to discharge, in the $(25 \mu \mathrm{g})$ fentanyl group compared with the (0) and $(10 \mu \mathrm{g})$ fentanyl groups Bindra et al. (2018).

In addition, Park et al. (2019) found increased duration of sensory block without prolonged motor blockade or recovery for ambulatory discharge with $(10 \mu \mathrm{g})$ fentanyl added to low dose $(5 \mathrm{mg})$ hyperbaric bupivacaine for knee arthroscopy.

All these findings are consistent with the present results that $(25 \mu \mathrm{g})$ fentanyl added to ultra-low dose $(2.5 \mathrm{mg})$ intrathecal bupivacaine neither increased the intensity of motor block nor prolonged the discharge time for anorectal surgery in the ambulatory setting.

In contrast to our findings, Gurbet et al. (2018) found significantly increased duration of sensory block with $(10 \mu \mathrm{g})$ intrathecal fentanyl added to $3 \mathrm{ml} 0.17 \%$ bupivacaine. This might be explained by protocol differences since the present study used ultra-low dose $(2.5 \mathrm{mg})$ intrathecal bupivacaine with $(25 \mu \mathrm{g})$ intrathecal fentanyl.

Comparing different doses of fentanyl (7.5, 10 and $12.5 \mu \mathrm{g}$ ) added to a fixed dose (5 mg, $0.17 \%$ ) of bupivacaine, Bhavya (2013) found that $12.5 \mu \mathrm{g}$ fentanyl provided better surgical anaesthesia and increased reliability of the block in minor urological procedures than (7.5) or (10 $\mu \mathrm{g})$ fentanyl.

As the spinal bupivacaine dose in the present study is lower than in that of Bhavya (2013) (25 $\mu \mathrm{g})$ fentanyl was used to provide longer sensory anesthesia without increasing discharge duration. The most consistent side-effect in the 
present study was pruritus in patients receiving intrathecal fentanyl, although in most cases it was mild and did not require treatment.

In other studies the side-effects of intrathecal fentanyl have been shown to be dose-related Ver Donck et al. (2014). Respiratory depression is a known complication of spinal opioids Orlov et al. (2013). This may be problematic with higher doses, as reported in a volunteer study Dahan et al. (2016).

In the present study, there were no clinical manifestations of respiratory depression with a fentanyl dose of (25 $\mu \mathrm{g}$ ). Additionally, Kumar et al. (2011) reported that $25 \mu \mathrm{g}$ intrathecal fentanyl in elderly patients did not lead to respiratory depression.

In the present study it was found that (25 $\mu \mathrm{g}$ ) intrathecal fentanyl reduced the analgesic requirement without increasing episodes of nausea or vomiting Pöpping et al. (2012). These findings were comparable with those of Zode and Dhumane (2015) who used (25 $\mu \mathrm{g})$ intrathecal fentanyl for lower extremity or genitourinary surgery, and Lee et al. (2011) who used $(0.5$ or $0.75 \mu \mathrm{g} / \mathrm{kg})$ intrathecal fentanyl for cesarean delivery.

\section{CONCLUSION}

Addition of $(25 \mu \mathrm{g})$ fentanyl to $(2.5$ $\mathrm{mg}) \quad 0.5 \%$ bupivacaine prolong the duration of sensory spinal block and reduced the analgesic requirement during the early post-operative period without increasing the incidence of opioid-related side-effects, except pruritus, or delaying hospital discharge in patients undergoing ambulatory anorectal surgery in comparison to using (5 $\mathrm{mg}) \quad 0.5 \%$ bupivacaine alone.

\section{REFERENCES}

1. Bhavya K (2013): A Comparative study of Low Dose Intrathecal Bupivacaine with Epidural Volume Expansion and Conventional Dose Spinal Anaesthesia in Caesarean Sections (Doctoral dissertation, Thanjavur Medical College, Thanjavur), 170(10):1134-42.

2. Bindra TK, Kumar P and Jindal G (2018): Postoperative analgesia with intrathecal nalbuphine versus intrathecal fentanyl in cesarean section: A double-blind randomized comparative study. Anesthesia, Essays and Researches; 12(2): 561-566.

3. Dahan A, Aarts L and Smith TW (2016): Incidence, reversal, and prevention of opioidinduced respiratory depression. Anesthesiology: The Journal of the American Society of Anesthesiologists, 112(1): 226-238.

4. Ferences $S$, Coloma $M$, White $P F$ and Chang LC (2012): Comparison of the costs and recovery profiles of three anesthetic techniques for ambulatory anal surgeries. Anesthesiology, 93: 1225 - 1230.

5. Gupta S, Sampley $S$, Kathuria $S$ and Katyal $S$ (2013): Intrathecal sufentanil or fentanyl as adjuvants to low dose bupivacaine in endoscopic urological procedures. Journal of Anesthesiology, Clinical Pharmacology; 29(4): 509-521.

6. Gurbet A, Turker G, Girgin NK, Aksu H and Bahtiyar NH (2018): Combination of ultra-low dose bupivacaine and fentanyl for spinal anaesthesia in out-patient anorectal surgery. Journal of International Medical Research; 36(5): 964-970.

7. Kendall MC, Alves LJC, Suh EI, McCormick ZL and De Oliveira GS (2018): Regional anesthesia to ameliorate postoperative analgesia outcomes in pediatric surgical patients: an updated systematic review of randomized controlled trials. Local and Regional Anesthesia, 11: 91-101.

8. Kumar B, Williams A, Liddle $D$ and Verghese M (2011): Comparison of intrathecal bupivacaine-fentanyl and bupivacaine- 


\section{COMBINATION OF ULTRA DOSES BUPIVACAINE PLUS FENTANYL...}

butorphanol mixtures for lower limb orthopedic procedures. Anesthesia, essays and researches; 5(2): 190-201.

9. Lee JH, Chung KH, Lee JY, Chun DH, Yang HJ, Ko TK and Yun WS (2011): Comparison of fentanyl and sufentanil added to $0.5 \%$ hyperbaric bupivacaine for spinal anesthesia in patients undergoing cesarean section. Korean Journal of Anesthesiology, 60(2): 103-119.

10. Maroof M, Khan RM, Siddique M, AND Charney DS (2015): Hypobaric spinal anesthesia with bupivacaine $(0.1 \%)$ gives selective sensory block for ano-rectal surgery. CanJAnaesth; 42: 691 - 694.

11. Martland, M. E., Rashidi, A. S., Bennett, M. I., Fallon, M., Jones, C., Rolke, R., and Mulvey, M. R: (2020): The use of quantitative sensory testing in cancer pain assessment: A systematic review. European Journal of Pain, 24(4), 669-684.

12. Maves TJ and Gebhart GF (2012): Antinociceptive synergy between intrathecal morphine and lidocaine during visceral and somatic nociception in the rat. Anesthesiology; 76: $91-99$.

13. Mehta N (2020): Adjuvant Drugs to Local Anesthetics. In Local Anesthetics. IntechOpen. 3-14.

14. Orlov D, Ankichetty S, Chung F and Brull R (2013): Cardiorespiratory complications of neuraxial opioids in patients with obstructive sleep apnea: a systematic review. Journal of Clinical Anesthesia, 25(7): 591-599.
15. Park, S. K., Lee, J. H., Yoo, S., Kim, W. H., Lim, Y. J., Bahk, J. H., and Kim, J. T. (2019): Comparison of bupivacaine plus intrathecal fentanyl and bupivacaine alone for spinal anesthesia with intravenous dexmedetomidine sedation: a randomized, double-blind, noninferiority trial. Regional Anesthesia \& Pain Medicine, 44(4), 459-465.

16. Pöpping DM, Elia N, Marret E, Wenk M and Tramèr MR (2012): Opioids added to local anesthetics for single-shot intrathecal anesthesia in patients undergoing minor surgery: a meta-analysis of randomized trials. PAIN $^{\circledR} ;$ 153(4): 784-793.

17. Sari, S., Sen, S., Kurt Omurlu, I., Oner Şavk, S., and Bakiş, M. (2015): Degree of motor block measured by bromage scale is not correlated with muscle relaxation. Enliven: J Anesthesiol Critical Care Med J, 2(2), 10051009.

18. Smith LE (2013): Ambulatory surgery for anorectal diseases: an update. South Med J,79: $163-166$.

19. Ver Donck A, Vranken JH, Puylaert M, Hayek S, Mekhail $\mathbf{N}$ and Van Zundert J (2014): Intrathecal drug administration in chronic pain syndromes. Pain Practice, 14(5): 461-476.

20. Zode A and Dhumane P (2015): Comparative evaluation of intrathecal bupivacaine plain versus intrathecal fentanyl plus bupivacaine in geriatric patients undergoing lower limb orthopaedic surgery. Research Chronicle in Health Sciences, 1(3): 183-192. 


\section{دراسة تأثّر مزج جرعات ثائقة الصفر من البيوبيفاكيز مع الثنثتانيل

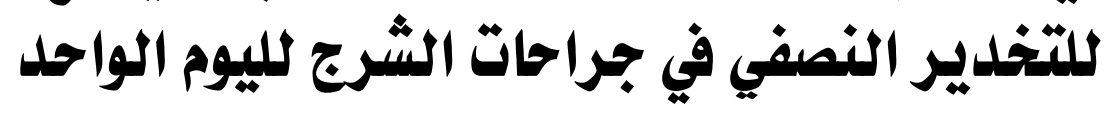

محمد صبحي ابراهيم احمد البحار, عبد الحافظ محمد الحسيني، احمد محمود محمد

$$
\text { الجارحي }
$$

قسم التخدير والرعاية المركزة، كلية الطب، جامعة الأزهر

E-mail: $\underline{\text { dr.ms.bahar3@gmail.com }}$

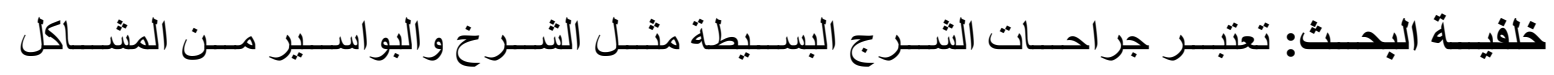

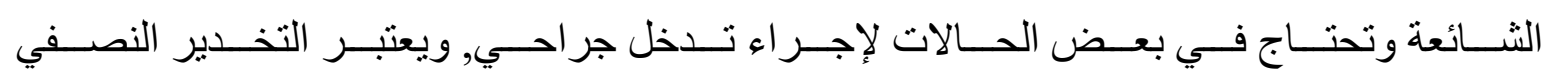
هو الإختيار الأمثل لمثل هذه الحالات.

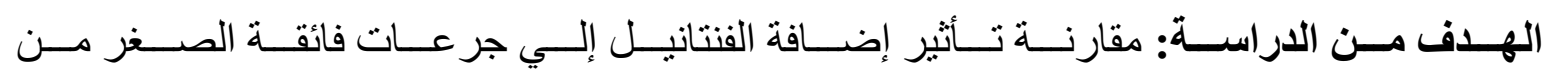
البيو بيفاكين في التخدير النصفي لعمليات جر احات الثرج البسيطة.

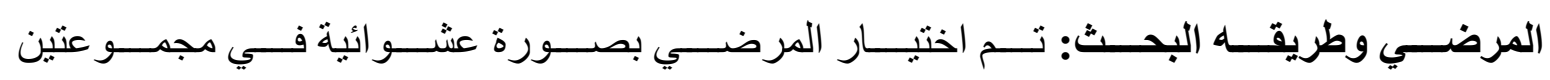
متساويتين:

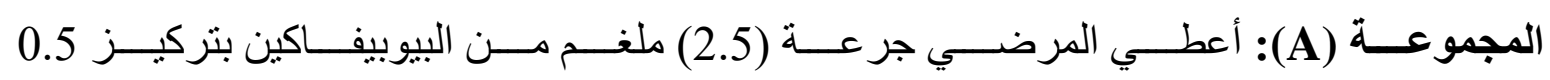
\% مع (25) ميكروجر ام من الفنتانيل.

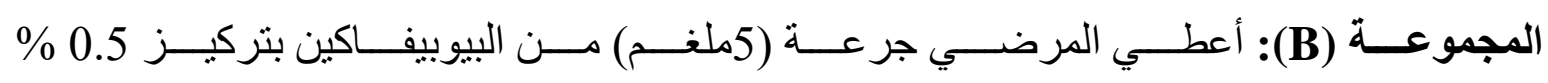
فقط.

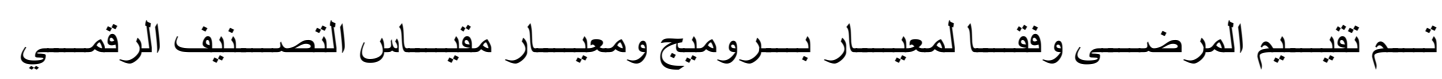

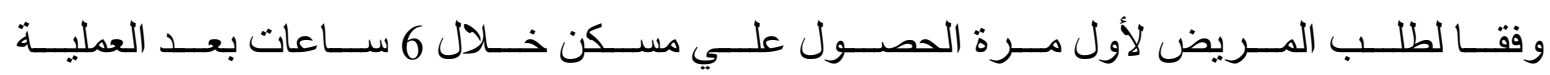

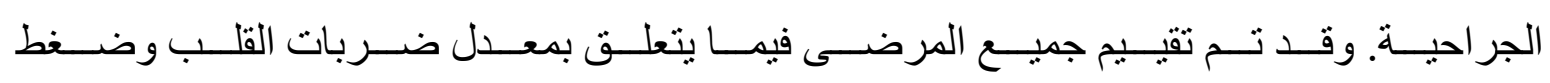

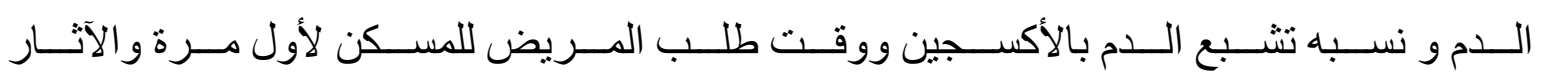

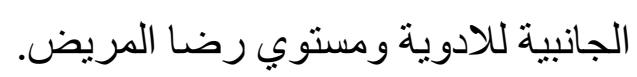

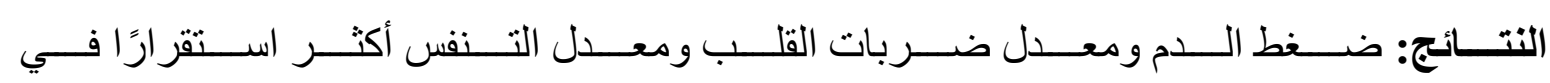

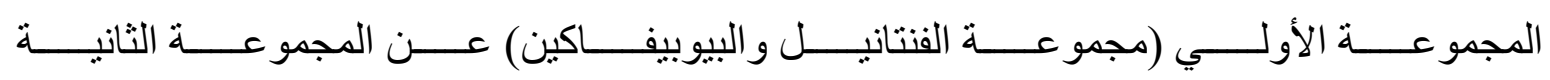




\section{COMBINATION OF ULTRA DOSES BUPIVACAINE PLUS FENTANYL... 1611}

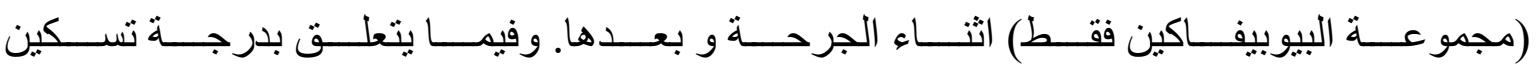

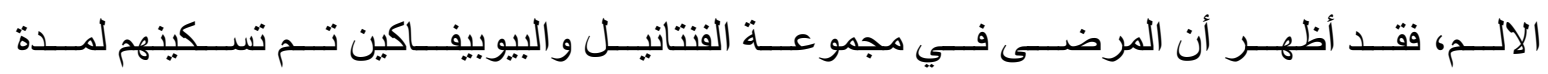

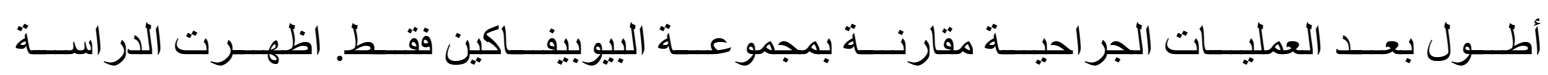

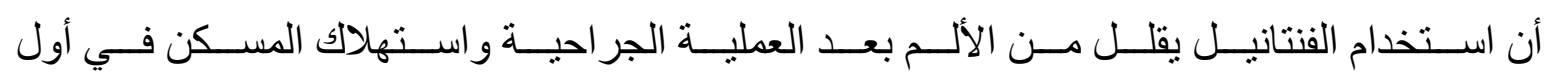

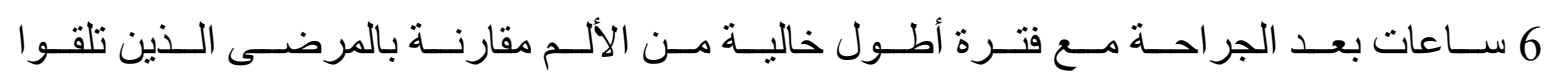
بيوبيفاكين فقط.

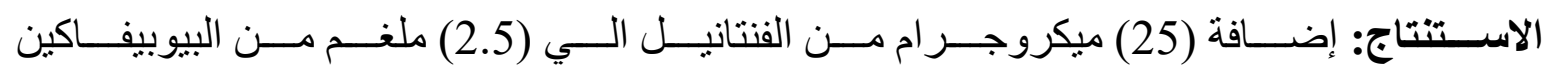

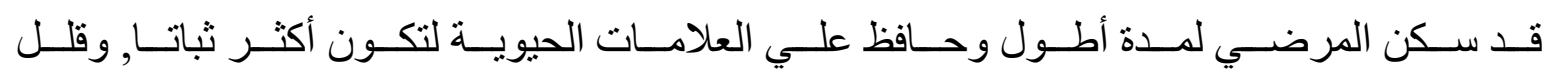

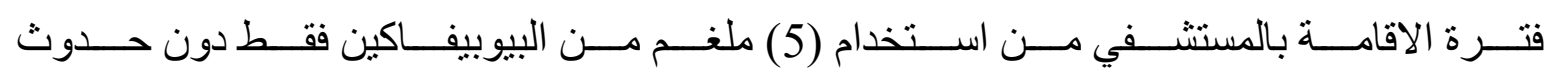
آثار جانبية باستثناء الهرش الذي اشتكي منه بعض المرضي. الكلمات الدالة: فنتانيل, بيوبيفاكين, جر احات الثرج. 